This document is the accepted manuscript version of the following article:

Miriyev, A., \& Kovač, M. (2020). Skills for physical artificial intelligence. Nature

Machine Intel1igence, 2(11), 658-660. https://doi.org/10.1038/s42256-020-00258-y

\title{
Skills for Physical Artificial Intelligence
}

\author{
Aslan Miriyev ${ }^{1,2, *}$, Mirko Kovac ${ }^{1,2, *}$ \\ ${ }^{1}$ Materials and Technology Center of Robotics, Empa- Swiss Federal Laboratories for Materials Science and \\ Technology, Überlandstrasse 129, 8600 Dübendorf, Canton of Zürich, Switzerland \\ ${ }^{2}$ Aerial Robotics Lab, Department of Aeronautics, Imperial College London, Exhibition Road, South Kensing- \\ ton Campus, London SW7 2AZ, UK \\ *Corresponding authors: aslan.miriyev@empa.ch ORCID: https://orcid.org/0000-0003-2110-2770; \\ , mirko.kovac@empa.ch,ORCID: https://orcid.org/0000-0002-9720-2463
}

\section{Synthesizing robots via physical artificial intelligence is a multidisciplinary challenge for future robotics research. An education methodology is needed for researchers to develop a combina- tion of skills in physical artificial intelligence.}

Akin to biological organisms, the next generations of robots are expected to act autonomously in the unstructured environment of the real world and be self-sustained in controller adaptation and learning ${ }^{1}$, physical resilience to damages ${ }^{2}$ and integration with collective systems ${ }^{3}$. These future robots will decide and adapt in real time on navigation, locomotion and manipulation by processing combinations of signals, simultaneously sent from multiple sensors in their "body" to their "brain". In addition, they will need to self-predict their capabilities ${ }^{4}$, self-heal ${ }^{5}$ and selfgrow $^{6}$ upon need, as well as maintain homeostasis ${ }^{7}$ in every situation to ensure harmonic balance between the task performance and self-preservation. Such intelligent behavior will result from a close interaction between the structure of the brain, body morphology and interaction with the environment. The developments in data-based, digital AI and learning capabilities in computation, algorithms and cognition have tremendously grown in the last decades, while the development of robots' "bodies", morphology and materials has lagged behind ${ }^{8-10}$. In the present paper, we introduce the physical counterpart of digital artificial intelligence, namely Physical Artificial Intelligence (PAI), and propose a methodology of educating the future generations of researchers to gaining skills for the creation of PAI.

\section{Physical Artificial Intelligence (PAI)}

Recent changes in human living have emphasized the need for remote and automated processes. However, today's robots are not mature and robust enough to perform everyday tasks, such as manipulating objects, or moving in unpredictable and complex environments, and are not sufficiently safe to interact with humans and natural outdoor environments. An appropriate balance 
between the brain and the body is a prerequisite for the creation of nature-like and fully integrated intelligent robots. Unlike robot design, which is to a large extent successfully automated by using software ${ }^{11}$, the design of novel materials and choice of development methods for robotics requires human contribution due to a broader combination of skills involved. For instance, in the emerging domain of soft robotics, there is still no systematic approach for choosing materials in combination with structural designs and controllers to obtain desired robot functionalities. Thus, one of the main gaps in robotics for the next decade is the development of materials ${ }^{12}$ and structures ${ }^{13,14}$ for robots' "bodies" and the co-evolution of body morphologies with intelligent control systems and learning-based methods ${ }^{15}$.

In order to bridge this gap, a strong trend in the robotics community is to co-evolve the body, control, morphology, actuation and sensing, which we name here as Physical Artificial Intelligence (PAI). PAI refers to the theory and practice of creating physical systems capable of performing tasks that are typically associated with intelligent organisms. The PAI methodology inherently combines the considerations of materials, design and manufacturing. Robots developed using PAI may autonomously perform tasks and maintain homeostasis in unstructured environments by exploiting the physical and computational features of their bodies along with the computational abilities of their "brains". Akin to biological organisms, PAI robotics can either replace digital AI or synergistically complement it by interfacing to a "brain". Many small robots, or those with limited computational functionality, would not include a dedicated central "brain" and their body's computation will guide their performance. Similar to the principles of natural diversity, PAI synthesis refers to robotic systems of any functionality, shape, size and habitat, with particular emphasis on integrating chemical, biological and material based functionalities. As such, PAI is independent of body variation approaches and distinguished from the embodied intelligence term ${ }^{9,16}$. The art of PAI synthesis is in creating successful combinations of various functional characteristics from the material level to morphology into robotic systems. PAI is illustrated in Fig. 1.

This paradigm of combining Physical Artificial Intelligence (PAI) and digital artificial intelligence is one of the most prominent frontiers in robotics development, offering a myriad of research opportunities. In particular, the integration of physical and computational robotic capabilities into a single approach of PAI may lead to a re-definition of the human-robot interaction by enabling the creation of symbiotic human-robot ecosystems. Currently, one of the main obstacles on this path is the lack of skills for PAI, with no coherent structure for education and training having been proposed. 


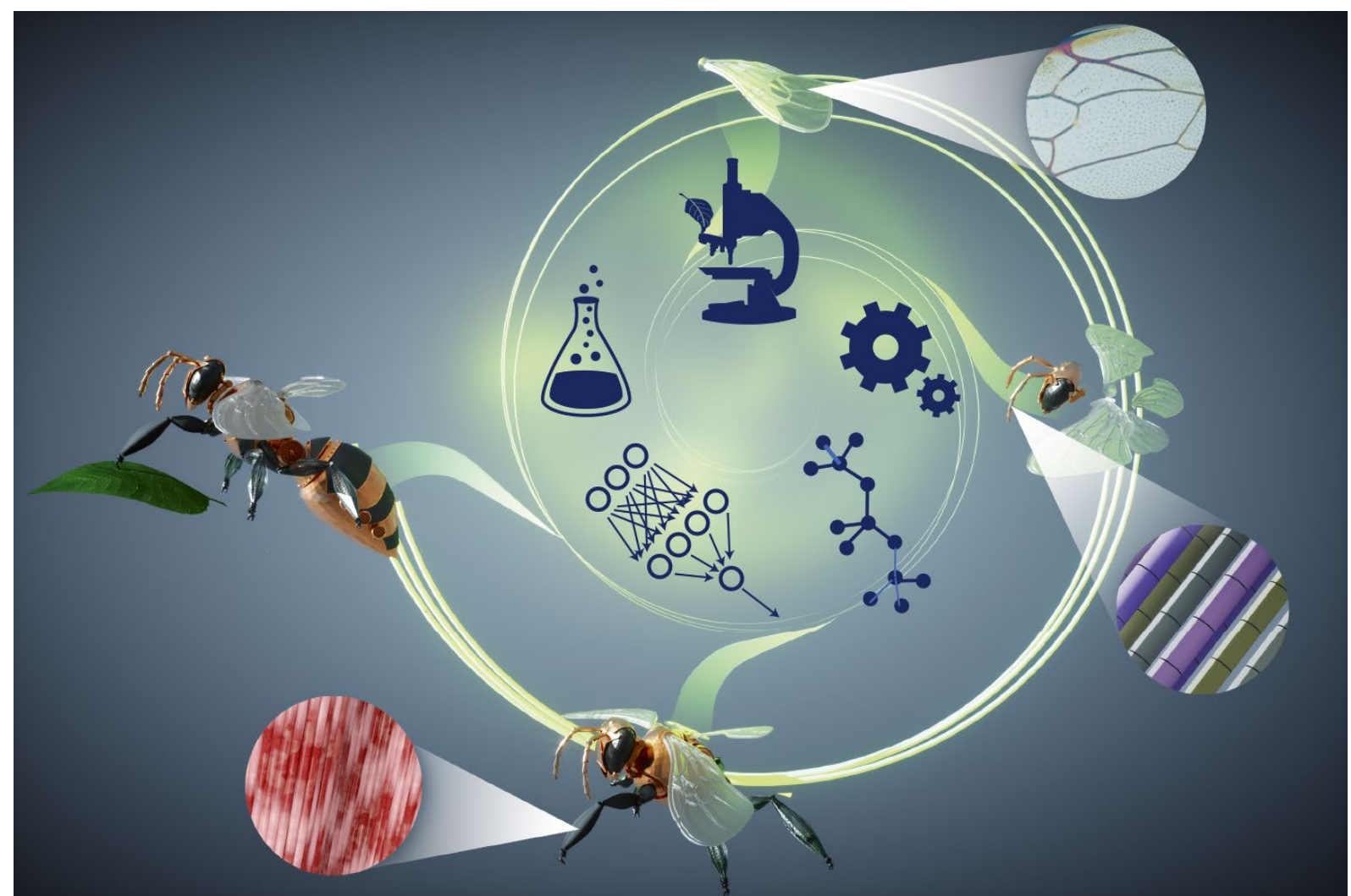

Fig. 1. PAl is the theory and practice of synthesizing nature-like intelligent robotic systems. This is achieved by using skills from multiple disciplines (materials science, mechanical engineering, computer science, chemistry and biology) to evolve a fully functional robot. The process is illustrated on a robotic bee that incorporates the considerations of materials, structure, self-perception and actuation, leading to the eventual culmination in a fully autonomous multi-functional robotic system. The disciplines feed into each of these evolution steps.

A number of disciplines are closely intertwined in the ambitious venture of creating

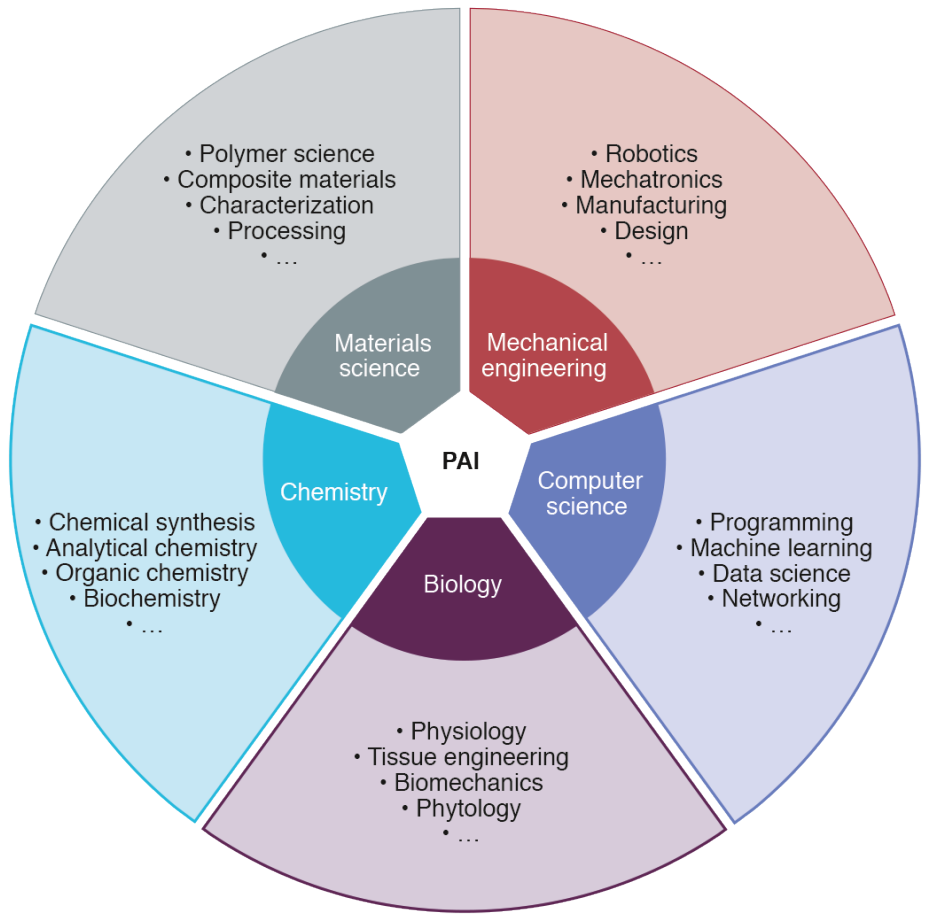

Fig. 2. Physical Artificial Intelligence (PAI) is comprised five main disciplines: materials science, mechanical engineering, computer science, biology and chemistry.
PAI. Mainly, these are materials science, mechanical engineering, computer science, chemistry and biology (Fig. 2). Leading the paradigm shift from robots as an assembly of hard devices ${ }^{17}$ to PAI-comprised robots requires a combination of skills in these disciplines. The domains shown for each discipline in Fig. 2 indicate the great plurality and diversity of the desirable skills, leaving space for even more. However, such a broad range of skills for a single person is difficult to obtain today, and no systematic educational approaches exist to develop them. 


\section{Developing skills for PAI}

We envision that acquiring multidisciplinary PAI skills requires a systematic educational approach on both the institutional and community level. As a part of this approach, we suggest the development of students' skills and support for mentors through the creation of a favorable institutional and community climate.

The following multi-level methodology can be proposed for implementing PAI in the current higher education programs. We suggest that the methodology needs to encompass the following three components: 1) institutional support, 2) appreciation of the complexity and challenges of working in interdisciplinary research, and 3) existence of venues for research recognition and dissemination.

First, support on the institutional level is vital for the ability to create a solid base for PAI skills. In our opinion, the main effort is required at the institutional level to explicitly encourage active cooperation in PAI between researchers from various departments and faculties. Institutional PAI clusters may create an educational platform, such as available multidisciplinary elective courses and laboratory training, and allow internal scientific collaborations and access to equipment. Importantly, properly functioning institutional PAI clusters will ensure professional guidance for choosing the correct combination of skills, fitting to the scope of interests or research needs of each student. This approach will also facilitate external collaborations between different institutional hubs, and foster national and international exchanges and extracurricular activities.

Due to its interdisciplinary nature, research in PAI benefits from a wider audience and the potential of publishing in higher impact journals. However, engagement in multidisciplinary research has a higher complexity due to the increased risks related to a wider professional expertise. Working between and within several disciplines requires courage to step out of comfort zones of terminology and networks, and necessitates continued learning along with a strong intrinsic motivation to do so. Moreover, career progression steps need to encourage and reward interdisciplinary work. For example, currently, new faculty members are often hired based on targeting specific narrow specialization areas rather than a multidisciplinary vision. It is vital to encourage interdisciplinary PAI research by recruiting faculty members whose expertise is in working at the nexus of PAI-related disciplines, along with providing appropriate institutional support. These steps will directly boost the PAI research, while returning multifold to the institution's prestige through high-impact publications, patents and attracted investments. 
In addition, it is important to promote venues for recognition and dissemination of multidisciplinary research. Belonging to a conventional scientific community, such as materials science, mechanics, organic chemistry or others, may narrow the presentation of a scientific problem to that which is "understandable" in the specific community. This approach lacks the vision of the whole and an understanding of the full complexity of the problem. The field of robotics and artificial intelligence, in particular, requires a wider, multi-disciplinary recognition model that emphasizes the complex interaction between disciplines and expertise. We propose to create and support communities of researchers, conferences and journals targeting the complex multidisciplinary challenges of PAI. Competitive fellowships, awards and prizes, co-organized by committees from different disciplines, may support the engagement on the community level.

\section{Conclusion}

We suggest that creating robots for a symbiotic human-robot ecosystem via the creation of physical artificial intelligence (PAI) will lead robotic research in the next decades. Currently, our community is missing the multidisciplinary skills for the creation of PAI, while most institutions still have not developed an infrastructure for such a challenging multidisciplinary effort. We proposed to advance the education of PAI skills by simultaneous development of multidisciplinary students' skills and support for mentors' activities by creating encouraging institutional and community environments. It is important to emphasize that the aim of the present communication is to highlight the importance of educating the next generation of researchers for creation of PAI, rather than to point to a precise ultimate guideline for action. We encourage a broad discussion on the topic in the scientific and research community and understand that different opinions and approaches may exist.

\section{References}

1. Cully, A., Clune, J., Tarapore, D. \& Mouret, J. B. Robots that can adapt like animals. Nature 521, 503-507 (2015).

2. Bilodeau, R. A. \& Kramer, R. K. Self-Healing and Damage Resilience for Soft Robotics: A Review. Front. Robot. AI 4, 48 (2017).

3. Petersen, K. H., Napp, N., Stuart-Smith, R., Rus, D. \& Kovac, M. A review of collective robotic construction. Science Robotics 4, (2019).

4. Xia, B. et al. Improving the Actuation Speed and Multi-Cyclic Actuation Characteristics of 
Silicone/Ethanol Soft Actuators. Actuators 9, 62 (2020).

5. Pena-Francesch, A., Jung, H., Demirel, M. C. \& Sitti, M. Biosynthetic self-healing materials for soft machines. Nat. Mater. 1-6 (2020). doi:10.1038/s41563-020-0736-2

6. Sadeghi, A., Mondini, A. \& Mazzolai, B. Toward self-growing soft robots inspired by plant roots and based on additive manufacturing technologies. Soft Robot. 4, 211-223 (2017).

7. Man, K. \& Damasio, A. Homeostasis and soft robotics in the design of feeling machines. Nat. Mach. Intell. 1, 446-452 (2019).

8. Sol, J. A. H. P. et al. Butterfly proboscis-inspired tight rolling tapered soft actuators. Chem. Commun. 55, 1726-1729 (2019).

9. Pfeifer, R., Bongard, J. \& Grand, S. How the body shapes the way we think: a new view of intelligence. (MIT Press, 2007).

10. Mengüç, Y., Correll, N., Kramer, R. \& Paik, J. Will robots be bodies with brains or brains with bodies? Sci. Robot. 2, 29 (2017).

11. Lipson, H. \& Pollack, J. B. Automatic design and manufacture of robotic lifeforms. Nature 406, 974-978 (2000).

12. Yang, G.-Z. et al. The grand challenges of Science Robotics. Sci. Robot. 3, eaar7650 (2018).

13. Kovac, M. Learning from nature how to land aerial robots. Science 352, 895-896 (2016).

14. Hauser, H. Resilient machines through adaptive morphology. Nat. Mach. Intell. 1, 338-339 (2019).

15. Howard, D. et al. Evolving embodied intelligence from materials to machines. Nat. Mach. Intell. 1, 12-19 (2019).

16. Chrisley, R. \& Ziemke, T. Embodiment. in Encyclopedia of Cognitive Science (John Wiley \& Sons, Ltd, 2006). doi:10.1002/0470018860.s00172

17. Miriyev, A., Stack, K. \& Lipson, H. Soft material for soft actuators. Nat. Commun. 8, 596 (2017). 